\title{
Le théâtre incarné, Etudes en hommage à Monique Dubar. Textes réunis par Franck Bauer et Guy Ducrey
}

\section{Luigi Luison}

\section{(2) OpenEdition Journals}

\section{Edizione digitale}

URL: http://journals.openedition.org/studifrancesi/35487

DOI: $10.4000 /$ studifrancesi.35487

ISSN: 2421-5856

\section{Editore}

Rosenberg \& Sellier

\section{Edizione cartacea}

Data di pubblicazione: 1 novembre 2005

Paginazione: 471-472

ISSN: 0039-2944

\section{Notizia bibliografica digitale}

Luigi Luison, «Le théâtre incarné, Etudes en hommage à Monique Dubar. Textes réunis par Franck Bauer et Guy Ducrey», Studi Francesi [Online], 146 (XLIX | II) | 2005, online dal 30 novembre 2015, consultato il 18 avril 2021. URL: http://journals.openedition.org/studifrancesi/35487 ; DOI: https:// doi.org/10.4000/studifrancesi.35487

Questo documento è stato generato automaticamente il 18 avril 2021.

\section{cc) (†) $\odot$}

Studi Francesi è distribuita con Licenza Creative Commons Attribuzione - Non commerciale - Non opere derivate 4.0 Internazionale. 


\title{
Le théâtre incarné, Etudes en hommage à Monique Dubar. Textes réunis par Franck Bauer et Guy Ducrey
}

\author{
Luigi Luison
}

\section{NOTIZIA}

AA. VV., Le théâtre incarné, Etudes en hommage à Monique DUBAR. Textes réunis par Franck BAUER et Guy DUCREY, Lille, Edition du Conseil Scientifique de l'Université Charles-de-Gaulle - Lille 3, 2003, pp. 270.

1 Il volume riunisce una serie di saggi dedicati a Monique DUBAR, grande storica del teatro europeo. L'intento degli autori è quello di mettere in evidenza i legami esistenti tra le diverse opere teatrali europee, analizzando anche altre arti della scena, come la danza.

2 Il testo, strutturato in quattro parti, si apre con il saggio di Joëlle PRUGNAUD (Le décor Moyen Âge sur la scène française au tournant du XIX ${ }^{\text {ème }}$ siècle, pp. 15-27). Attraverso le opere di Maeterlinck e Claudel, l'autore evidenzia l'interesse degli scrittori post romantici per l'epoca medievale, sottolineando come la rappresentazione del Medioevo sulla scena segni un cambiamento radicale nella Francia del 1880, nell'ambito dell'organizzazione dello spazio scenico.

3 Fiona MCINTOSH-VARJABÉDIAN (Cromwell, figure dramatique et figure historique, pp. 29-39) mette a confronto Cromwell di Victor Hugo e l'Histoire de la Révolution d'Angleterre di Guizot per dimostrare come la figura del personaggio principale è stata trattata a partire dalle fonti comuni. 

pp. 41-55) effettua un percorso che esalta la maestria di Rostand nel mettere in piedi un Cyrano immaginario e nello stesso tempo estremamente coerente. Strindberg lancia con la sua pièce Mademoiselle Julie del 1888 ai drammaturghi contemporanei quali Ibsen, Cechov e Hauptmann; provocazione che consiste nel privilegiare come luogo dell'azione uno spazio non aperto al pubblico, la cucina.

Karl ZIEGER (Arthur Schnitzler et le naturalisme: Das Märchen à l'épreuve de la dramaturgie naturaliste, pp. 65-76) vuole mostrare come l'opera di Schnitzler si inscrive nella drammaturgia naturalista, evidenziando, in particolare, il ruolo che il drammaturgo austriaco attribuisce al teatro come luogo sociale.

7 Paul RENARD (La figure de l'ouvrier dans le théâtre de combat, pp. 77-82) disegna un ritratto preciso della scena teatrale tra il 1896 e il 1911; periodo che vede il teatro farsi vieppiù impegnato, e dare particolare risonanza al proletariato maschile.

Jean-Marc MOURA (Danser l'orient. De Petipa à Noureev: le durable prestige de La Bayadère, pp. 83-89). Il balletto ci introduce al gusto dell'esotismo attraverso l'evoluzione de $L a$ Bayadère. L'autore illustra come la trasposizione dell'Oriente sul palcoscenico abbia sedotto i maggiori scrittori europei del XIX secolo.

Jacques BOULOGNE (Le déplacement du sacré dans le théâtre d'Anouilh, pp. 93-109) confronta il drammaturgo francese con i suoi predecessori antichi, Sofocle ed Euripide. Attraverso il confronto tra le due pièce di Anouilh, Antigone e Médée, l'autore mostra come nei testi del drammaturgo francese non si possa parlare di dissacrazione, ma di spostamento del sacro, nella misura in cui l'aspirazione all'assoluto passa dal rispetto della pietà filiale al rispetto di sé.

Marie-Madeleine CASTELLANI (Du roman au théâtre: La fille du roi de Hongrie, Miracle de Notre Dame par personnages, pp. 111-125) parla della trasposizione del romanzo in forma teatrale. In particolare viene preso in esame il romanzo di Philippe de Rémi, La Manekine, portato sulla scena teatrale sotto forma di Miracles de Notre Dame. Degni di nota sono i rimaneggiamenti subìti sino ai primi del Novecento.

11 Franck BAUER (Molière, Dancourt: l'école de Marivaux. Contribution au dénouement d'une intrigue intertextuelle, pp. 127-142). Da L'École des mères di Marivaux parte lo studio dell'autore che, attraverso parallelismi con L'École des femmes di Molière, mette in evidenza numerose similitudini anche con la pièce teatrale di Dancourt del 1691 dal titolo L'École des maris.

Michèle HECQUET (Sand et Sedaine: Le mariage de Victorine - 1851, pp. 143-152). La pièce in tre atti di George Sand persegue e radicalizza la sua innovazione drammatica in favore di un teatro intimo, interiore.

13 Chantal Liaroutzos (Olivier de Serres, ou l'agriculture comme théâtre, pp. 155-163). La riflessione dell'autore ruota attorno al Théâtre d'Agriculture et mesnage des champs di Olivier de Serres, il primo manuale di agricoltura che riporta, nel titolo, il termine Théâtre. Questo testo presenta una riflessione consapevole sui modi di rappresentare il reale. Il suo successo risiede non solo in un perfetto adeguamento di una poetica e di un sapere pratico, ma soprattutto nel fatto che la poetica è produttrice di concetti sul piano del sapere pratico in questione.

Studi Francesi, 146 (XLIX | II) | 2005 
14 Anne DUCREY (Au pays des oiseaux bleus, Maeterlinck ou le voyage en allégorie, pp. 165-176). L'Oiseau bleu, composto da Maeterlinck nel 1906, apre, secondo l'autrice, una riflessione che conferisce al testo un aspetto singolare, che oscilla tra incantesimo e favola morale. Attraverso pagine autenticamente simboliste, Maeterlinck mette in scena l'invisibile rivelato dal visibile, grazie all'allegoria.

15 Jacques LANDRECIES (De la marionette à l'opérette: le théâtre en picard de l'Amiénois Camille Dupetit, pp. 177-192) presenta il teatro dialettale in piccardo di Camille Dupetit. Erede del lavoro iniziato da Edouard David negli ultimi anni dell'Ottocento, Dupetit perfeziona e personalizza il passaggio dal teatro delle marionette alla scena vera e propria, unendo al dialetto e al francese la musica e il canto.

16 Guy DUCREY (Les bateaux-théâtres de Jean Cocteau, pp. 193-204). La particolare metafora marittima che Cocteau adatta al suo universo e che sembra investita da un'alta valenza poetica, prende il valore, secondo l'autore, di una professione di fede poetica che consiste nell'allontanare dalla creazione tutto ciò che è fragile per essere visto da lontano, e costruire, invece, una robusta macchina in grado di affrontare tutte le tempeste.

Dimitri SOENEN («Sauve qui peut/et persone ne peut se sauver» ou le spectacle intolérable du monologue, pp. 205-216) illustra le principali caratteristiche della pièce-monologo e descrive l'atteggiamento del pubblico europeo del XX secolo nell'assistere a questo tipo di spettacolo.

18 Pierre BRUNEL (Claudel et Kafka. À propos d'une mise en scène du Procès, pp. 219-234) analizza la trasposizione teatrale, da parte di Claudel, del romanzo di Kafka, il Processo. Lo spettacolo, tenutosi nel 1947, ha dato modo a Claudel di riflettere sul complesso giudeo-cristiano; una situazione vissuta in anticipo dalla Storia e che nel tempo si è radicalizzata.

Michel AUTRAND (L'anarchisme au féminin dans le théâtre de Claudel: le personnage de Lâla, pp. 235-242) effettua un accurato studio sull'anarchismo propriamente femminile. L'analisi effettuata sui personaggi femminili di Thalie e Lâla contribuisce a sfumare il modo in cui Claudel vede il femminile.

Anne-Rachel HERMETET (Le Gorille catholique: Claudel en Italie dans l'entre-deux-guerres, pp. 243-253) espone la posizione di Claudel nei riguardi di una polemica più religiosa che letteraria scoppiata in Italia negli anni trenta. Gli scritti di Claudel si inseriscono proprio nella confluenza delle due principali problematiche: la prima, che riguarda la letteratura religiosa, ovvero la possibilità di fondare un'arte cattolica, e la seconda che interessa le rappresentazioni delle nuove forme drammatiche del teatro moderno.

21 Il volume si chiude, infine, con il saggio di Aimé PETIT (Les premiers éclats de «vitre» dans la littérature française $d u$ Moyen Âge, pp. 257-265) sulla prima comparsa della parola « vitre » nella letteratura francese del Medioevo e sulle varie accezioni che tale termine ha assunto nei romanzi dell'epoca. 